\title{
Ultrasound-Guided Lumbar Spine Injection for Axial and Radicular Pain: A Single Institution Early Experience
}

\author{
Matthew Tay ${ }^{1,2}$, Shauna Christine Sim Hwei Sian ${ }^{1}$, Chen Zhi Eow ${ }^{3}$, \\ Kelvin Lor Kah $\mathrm{Ho}^{4}$, Joo Haw $\mathrm{Ong}^{1}$, Dinesh Sirisena ${ }^{1}$ \\ ${ }^{1}$ Sports Medicine Centre, Khoo Teck Puat Hospital, Singapore \\ ${ }^{2}$ Department of Rehabilitation Medicine, Tan Tock Seng Hospital Rehabilitation Centre, Singapore \\ ${ }^{3}$ Yong Loo Lin Medical School, National University of Singapore, Singapore \\ ${ }^{4}$ Department of Orthopaedic Surgery, Khoo Teck Puat Hospital, Singapore
}

Study Design: Clinical audit via retrospective review of a database.

Purpose: To report an early experience using ultrasound-guided lumbar spinal injection for axial and radicular pain in an Asian multiethnic cohort.

Overview of Literature: Ultrasound-guided spine injection therapy is a comparatively new technique in the management of axial and radicular pain from degenerative lumbar spinal conditions, which may be a reasonable alternative to conventional fluoroscopic or computed tomography-guided injection.

Methods: A retrospective review was conducted, involving all patients who underwent ultrasound-guided lumbar spine injection therapy at a single institution over 1 year. Patients were evaluated by two interventionists, who then performed standardized ultrasound-guided lumbar facet joint and pararadicular spinal injections.

Results: There were 42 patients treated at our Sports Medicine Centre; with 27 patients $(64.3 \%)$ receiving facet joint injections and 18 patients (42.9\%) receiving nerve root injections. The majority $(90.5 \%)$ of patients experienced an improvement of $>30 \%$ in pain intensity at 3 months post-injection, using the Numerical Rating Scale pain score $(p<0.001)$; with 40 patients $(95.2 \%)$ reporting a reduction in Oswestry Disability Index score $(p<0.001)$. No complications were reported.

Conclusions: Our initial experience confirms the safety, feasibility, and effectiveness of ultrasound-guided lumbar spinal injection for the treatment of axial and radicular pain in an Asian multiethnic cohort.

Keywords: Lumbosacral region; Back pain; Spinal injections; Ultrasonography

\section{Introduction}

Low back pain (LBP) is a common cause of disability in the adult population, with a lifetime prevalence as high as
84\% [1]. Chronic LBP, defined as pain persisting beyond 3 months, may result in a prolonged loss of function, negative economic impact, including loss of work productivity, and large treatment costs [2].

Received Dec 29, 2019; Revised Mar 7, 2020; Accepted Mar 15, 2020

Corresponding author: Matthew Tay

Department of Rehabilitation Medicine, Tan Tock Seng Hospital Rehabilitation Centre, 17 Ang Mo Kio Avenue

9, 569776, Singapore

Tel: +65-64506164, Fax: +65-64590414, E-mail: matthew_rj_tay@ttsh.com.sg 
Pain may arise from numerous anatomical structures in the lumbar spine, including zygapophyseal (facet) joints, nerve roots, disks, and sacroiliac joints (SIJs). Of these structures, facet joint syndrome, radicular pain, and SIJ pain are common culprits of spinal pain. Lumbar facet joints, which are highly innervated, can develop arthritic changes or inflammation [3] and have been implicated as a source of pain in up to $45 \%$ of patients with LBP [4]. Inflammation or compression of the spinal nerve root is believed to contribute to radicular pain, with disk herniation being the most common cause [5]. Though underdiagnosed, SIJ pain is also increasingly recognized as a source of LBP, affecting $15 \%-30 \%$ of patients with non-radicular pain arising from intra- or extra-articular sources [6]. Identification of pain generators often requires careful assessment, examination, and diagnostic imaging.

Apart from rehabilitative approaches, psychotherapy, and pharmacology, injection therapies targeted to the nerve roots and facet joints play a major role in a multidisciplinary pain management approach [7]. The use of imaging guidance with computed tomography (CT) or fluoroscopy in facet joint, pararadicular, or periradicular injections, is well-established and currently used widely. On the other hand, ultrasound-guided spine injection therapy is a relatively new, but promising, technique that has been shown to be reliable and accurate [8], and it does not require exposure to ionizing radiation or expensive equipment and facilities. Various techniques for ultrasound-guided spine injection have previously been described [9]. For example, lumbar facet pain can be addressed via medial branch blocks [10] or intra-articular facet injection [11]; while radicular pain can be treated via caudal epidural injection [12], interlaminar epidural injection [13] or transforaminal injection [14,15]. Many of these ultrasound-guided spine injection techniques have been validated via cadaveric, fluoroscopic, and CT studies with reports of statisfactory needle placement, a good safety profile, and immediate post-injection pain improvement [9]. However, there is limited literature investigating sustained pain relief and disability reduction in such patients undergoing ultrasound-guided spine injection therapy, and studies also differ in techniques used. Although outcomes for medial branch blocks and caudal epidural injection have been published [16,17], literature on facet joint and pararadicular injection is limited $[18,19]$. This is even though facet joint and pararadicular injection may be more reliable than previously described techniques, in terms of ultrasound localization and pain reduction, respectively $[9,20]$.

An ultrasound-guided spinal injection service was recently introduced at a public hospital in Singapore, and a clinical audit of patients undergoing ultrasound-guided pararadicular and facet joint injection for LBP was conducted to determine the feasibility and efficacy of this procedure in producing sustained pain relief and disability reduction.

\section{Materials and Methods}

This study is a retrospective clinical audit of all patients who underwent ultrasound-guided lumbar spinal injection for chronic axial and radicular pain in an acute public hospital sports medicine center between June 1,2018 and June 1, 2019. All patients were either referred by other clinical specialties, or by primary care physicians. Inclusion criteria for this audit were patients who were $\geq 21$ years old, had clinical-radiologic signs of axial LBP or radicular pain for $\geq 3$ months, had failed conservative treatment, and had current lumbar spine $\mathrm{CT}$ or magnetic resonance images available. Excluded from analysis were patients with pregnancy, spinal tumors, focal paresis, symptomatic lumbar stenosis, anticoagulation drug therapy/coagulopathy, and psychiatric conditions likely to undermine the diagnostic workup or treatment response. This clinical audit was performed in accordance with the principles of the Declaration of Helsinki. The requirement of informed consent for patient inclusion was waived by the National Healthcare Group Domain Specific Review Board.

Patients were evaluated by two sports physicians with experience in musculoskeletal ultrasound, and the levels for spinal injection were selected based on standard diagnostic and clinical methods, including physical examination, referral patterns, and imaging [21]. A standardized ultrasound-guided lumbar approach was utilized for facet and pararadicular spinal injections [11]. The appropriate spinal level was first defined in the long-axis midline view, starting from the sacrum toward the cephalad spinous processes. In ultrasound-guided pararadicular spinal injections, the transducer was then translated laterally at the respective spinal segment in a parasagittal orientation, until the transition from the vertebral arch to the facet joints was reached. The transducer was then translated further until the transverse processes were seen and then back toward the midline until the edge of the facet joints were 
seen (Fig. 1). In this scanning plane, the intertransverse ligament was seen as a thin, hyperechoic band between two adjacent transverse processes, with the corresponding spinal nerve (which may, or may not, have been visualized) underneath the ligament. A needle was then inserted using an in-plane technique, until the targeted segmental intertransverse ligament was penetrated by the needle tip, which indicated that the needle was in the pararadicular segment (Fig. 1).

In ultrasound-guided facet joint injections, the transducer was rotated into a short-axis view over the target spinous process and moved laterally to the respective facet joint (Fig. 2). The lamina of the vertebral arch, facet joint, inferior articular process, and mammillary process were then delineated. A needle was then inserted laterally from the midline, lateral to the transducer, using an in-plane technique, which allowed visualization of the needle path (Fig. 2). Interventions were performed on a standardized ultrasound machine (LOGIQ P5; General Electric, Boston, MA, USA) using a broad range curved 5-1 MHz or a 7-12 MHz linear array transducer, depending on the patient's body mass.

For facet joint injections, $2 \mathrm{mg}(0.5 \mathrm{~mL})$ of dexamethasone or triamcinolone acetate and $1.5 \mathrm{~mL}$ of $1 \%$ lidocaine were injected into the facet joint once the needle was positioned correctly under ultrasound guidance. For pararadicular compartment injections, $2 \mathrm{mg}(0.5 \mathrm{~mL})$ of dexamethasone was used instead. Depending on the patient's body mass, either a 20G spinal needle (20G, 8.89 $\mathrm{cm}, 0.9 \times 88 \mathrm{~mm}$; B. Braun, Melsungen, Germany), or conventional needle (21G, $7.93 \mathrm{~cm}, 0.8 \times 80 \mathrm{~mm}$; B. Braun) was used.
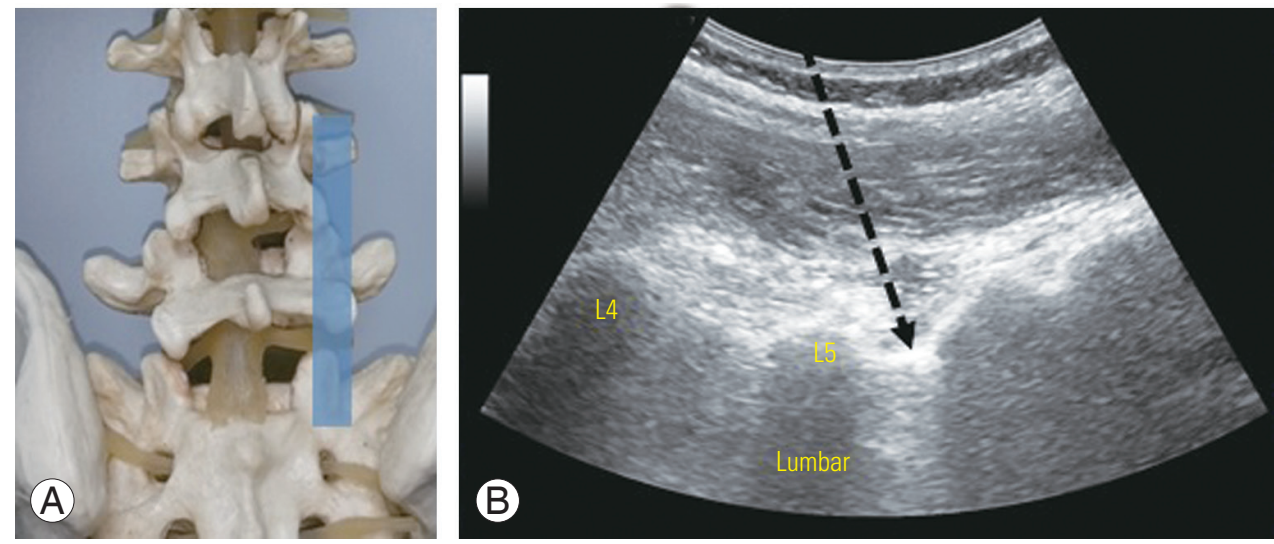

Fig. 1. (A) Spine model with intended ultrasound window. (B) Ultrasound view of the posterior sagittal paravertebral plane demonstrates ultrasound-guided lumbar spine pararadicular nerve root injection. The intended needle placement (arrow) is performed in an in-plane direction, with the needle tip penetrating the thin hyperechoic intertransverse ligament.
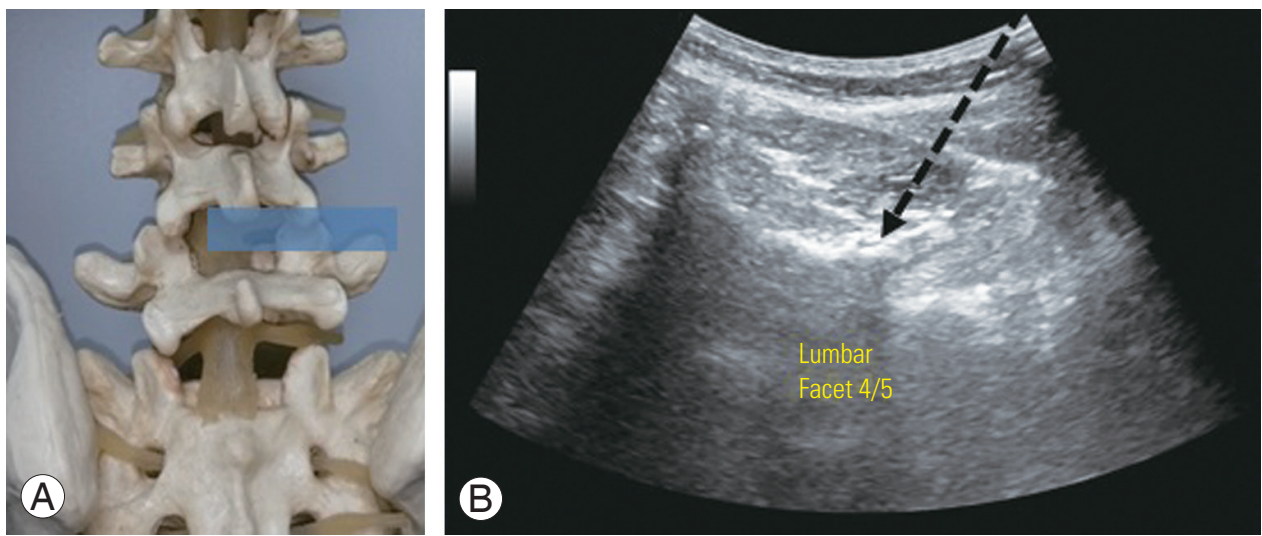

Fig. 2. (A) Spine model with intended ultrasound window. (B) Ultrasound view of the posterior transverse paravertebral plane demonstrates ultrasound-guided lumbar spine facet joint injection. The intended needle placement (arrow) is performed in an in-plane direction. 
Baseline demographic information, clinical variables, use of oral analgesia, and adverse events were collected from medical records or patient interview. The primary outcome of pain was measured based on a 11-point $\mathrm{Nu}$ merical Rating Scale (NRS), with 0 indicating no pain and 10 indicating the worst pain imaginable. This was determined prior to the injection, at 1 week, 1 month, and 3 months post-injection. A 2-point or more decrease or $30 \%$ reduction on the NRS were deemed to represent clinically meaningful improvements in pain intensity [22]. The Oswestry Disability Index (ODI) score was recorded 3 months post-injection. The ODI is a measure of functional status based on a patient-completed questionnaire, with a score of 0 indicating no restriction of daily activities and 100 indicating extreme disability [23].

Statistical analyses were generated using the IBM SPSS statistics ver. 23.0 (IBM Corp., Armonk, NY, USA). Descriptive statistics were utilized to illustrate patient demographics and clinical characteristics. Changes in outcomes from baseline at 1 week, 1 month, and 3 months were evaluated using paired-sample $t$-tests. A $p$-value of $<0.05$ was considered statistically significant for a two-tailed test.

\section{Results}

A total of 42 patients, treated consecutively at the sports medicine center, were identified. The average age of the audited population was $46.6 \pm 19.0$ years old, with the majority being male patients (66.7\%). The demographic profile and clinical characteristics are shown in Table 1. Ten patients received bilateral facet joint and/or nerve root injections, and three patients received combined nerve root and facet joint injections.

Positive outcomes were seen at 3 months post-injection, with 36 patients ( $85.7 \%$ ) reporting a $\geq 2$ point reduction in NRS pain score and 38 patients (90.5\%) reporting a $>30 \%$ reduction in NRS pain score. There were 40 patients (95.2\%) who reported a reduction in ODI score; with an improved 3-month post-injection average ODI score of $31.9 \pm 18.0$, compared to the pre injection baseline average ODI score of $53.2 \pm 12.6$. This difference was statistically significant $(p<0.001)$ (Table 2).

There were no adverse effects documented for these patients, and none reported worsening pain intensity on the NRS over a 3-month follow-up. None required a repeated spinal injection, and no patients were subsequently sched-
Table 1. Demographic characteristics of patients with low back pain ( $N=42$

\begin{tabular}{|c|c|}
\hline Characteristic & Value \\
\hline Age (yr) & $46.6 \pm 19.0$ \\
\hline Male & $28(66.7)$ \\
\hline \multicolumn{2}{|l|}{ Ethnicity } \\
\hline Chinese & $26(62.0)$ \\
\hline Malay & $9(21.4)$ \\
\hline Indian & $7(16.7)$ \\
\hline Married & $25(59.5)$ \\
\hline Smoker & $13(31.0)$ \\
\hline \multicolumn{2}{|l|}{ Education level (yr) } \\
\hline$\leq 6$ & $15(35.7)$ \\
\hline $7-12$ & $22(52.4)$ \\
\hline$>12$ & $5(11.9)$ \\
\hline \multicolumn{2}{|l|}{ Occupation } \\
\hline Working & $24(57.1)$ \\
\hline Not working & $18(42.9)$ \\
\hline \multicolumn{2}{|l|}{ Inciting event } \\
\hline Fall & $10(23.8)$ \\
\hline Work related & $10(23.8)$ \\
\hline Sports & $3(7.1)$ \\
\hline None & $19(45.2)$ \\
\hline \multicolumn{2}{|l|}{ Analgesic use immediately post-injection } \\
\hline NSAID usage & $8(19.0)$ \\
\hline Opioid usage & $5(11.9)$ \\
\hline \multicolumn{2}{|l|}{ Analgesic use at 6 months post-injection } \\
\hline NSAID usage & $6(14.3)$ \\
\hline Opioid usage & $2(4.76)$ \\
\hline Duration of pain (mo) & $9.19 \pm 6.51$ \\
\hline Baseline Numerical Rating Scale pain score in the past week & $6.19 \pm 1.73$ \\
\hline Baseline Oswestry Disability Index score & $53.2 \pm 12.6$ \\
\hline Body mass index $\left(\mathrm{kg} / \mathrm{m}^{2}\right)$ & $22.6 \pm 3.36$ \\
\hline \multicolumn{2}{|l|}{ Site of injection } \\
\hline Bilateral & $10(23.8)$ \\
\hline Left & $19(45.2)$ \\
\hline Right & $13(31.0)$ \\
\hline Patients with nerve root injections & $18(42.9)$ \\
\hline Patients with facet joint injections & $27(64.3)$ \\
\hline Levels injected for each patient & $1.40 \pm 0.627$ \\
\hline
\end{tabular}

Values are presented as mean \pm standard deviation or number $(\%)$. NSAID, nonsteroidal anti-inflammatory drugs. 
Table 2. Outcomes of patients who had undergone injection for low back pain

\begin{tabular}{|c|c|c|}
\hline Variable & Outcome ( $n=42)$ & $p$-value \\
\hline \multicolumn{3}{|l|}{1 Week post-injection } \\
\hline NRS pain score & $3.48 \pm 2.19$ & $<0.001^{\text {al }}$ \\
\hline Reduction in NRS pain score from baseline & $2.71 \pm 1.82$ & NA \\
\hline Patients with reduction in NRS pain score by $\geq 2$ & $27(64.3)$ & NA \\
\hline Patients with reduction in NRS pain score by $>30 \%$ & $27(64.3)$ & NA \\
\hline \multicolumn{3}{|l|}{1 Month post-injection } \\
\hline NRS pain score & $3.60(2.19)$ & $<0.001^{\mathrm{a})}$ \\
\hline Reduction in NRS pain score from baseline & $2.60(1.94)$ & NA \\
\hline Patients with reduction in NRS pain score by $\geq 2$ & $25(59.5)$ & NA \\
\hline Patients with reduction in NRS pain score by $>30 \%$ & $25(59.5)$ & NA \\
\hline \multicolumn{3}{|l|}{3 Months post-injection } \\
\hline NRS pain score & $2.29(1.37)$ & $<0.001^{\text {a) }}$ \\
\hline Reduction in NRS pain score from baseline & $3.90(1.87)$ & NA \\
\hline Patients with reduction in NRS pain score by $\geq 2$ & $36(85.7)$ & NA \\
\hline Patients with reduction in NRS pain score by $>30 \%$ & $38(90.5)$ & NA \\
\hline ODI score & $31.9 \pm 18.0$ & $<0.001^{\text {b) }}$ \\
\hline Reduction in ODI from baseline & $21.4 \pm 13.8$ & NA \\
\hline
\end{tabular}

Values are presented as mean \pm standard deviation or number (\%).

NRS, Numerical Rating Scale; NA, not applicable; ODI, Oswestry Disability Index.

${ }^{a}$ Compared to baseline NRS score. ${ }^{\text {b) }}$ Compared to baseline ODI score.

uled for, or required, spine surgery during follow-up at 6 months post-injection.

\section{Discussion}

Improved outcomes after spinal injection have been demonstrated in patients with LBP secondary to facet joint syndrome and radicular pain from nerve root compression. Steroids are commonly used in these local injections to address inflammatory pathways implicated in the LBP pathogenesis. Image guidance is also routinely used by pain interventionists to reduce neurovascular complications and to improve delivery of medications to the intended target [24]. Although fluoroscopic or CT guidance are currently the most well-established modalities, they pose the risk of ionizing radiation exposure to the patient and the operator. Radiation doses received by the patient have been reported to be as high as $8,992 \mu \mathrm{Gy} \cdot \mathrm{m}^{2}$ per procedure, depending on the technique and image guidance used [25]. Ultrasound guidance, on the other hand, does not pose any radiation exposure risk. Apart from the lack of ionizing radiation, benefits of ultrasound guidance include direct visualization of the target of interest, visualization of the spread of local anesthetics, reduced intervention time, decreased complications caused by needle malposition, and a reduced amount of local anesthetic required $[8,11]$. Importantly, comparative trials between ultrasound and CT or fluoroscopic guidance have shown similar accuracy and efficacy $[8,12-14]$. In patients with intractable LBP, ultrasound-guided spinal injection also presents a less invasive and less morbid alternative to surgery $[8,9]$. This may be particularly relevant for patients who are medically unfit or have a higher risk of surgical and anesthetic complications.

This clinical audit demonstrates that ultrasound guidance is a safe and clinically efficacious modality for spinal injection. Although some Western data suggests ethnic differences in back pain [26], nearly all of the patients in our multiethnic cohort reported an improvement in their pain intensity scores after the injection. Notably, the improvement in pain was also sustained at 1 and 3 months post-injection. This was also closely linked with a lower ODI index score at 3 months, suggesting that significant functional outcomes can be achieved with the reduction 
in pain achieved from spinal injection. Although most studies on ultrasound-guided spine injection therapy report an immediate therapeutic effect [9], our study adds to the limited literature by demonstrating a sustained benefit with an associated improvement in functional status. The reduction in NRS and ODI outcomes post-injection by $3.90 \%$ and $22.4 \%$, respectively, are also similar to that reported in a systematic review by Hofmeister et al. [27]. This suggests that the interventionists in this audit were able to successfully identify and treat their patients' predominant pain generators. This audit also demonstrates that more than one pain contributor may be present in LBP [28], as 10 patients received bilateral facet joint and/ or nerve root injections, and three patients received combined nerve root and facet joint injections.

The heterogeneity of post-procedure responses supports the fact that LBP remains a diagnosis with multiple psychosocial and neurophysiological factors. Although focused on short-term outcomes, interventionists must often accept the fact that interventions can modulate, but not necessarily cure, the underlying pathology, and realistic expectations of treatment should be communicated to patients. Furthermore, invasive spinal procedures should not be the sole treatment modality. Patients with chronic LBP often have coexisting psychosocial issues, and so a multidisciplinary approach, with the integration of psychosocial, rehabilitative, complementary, and pharmacological modalities, is needed to treat chronic LBP effectively.

Despite its many advantages, there are several drawbacks to the use of ultrasound guidance in spinal injection. Ultrasound-guided spinal injection remains technically challenging and requires a steep learning phase [9]. As it permits a smaller field of vision compared to CT or fluoroscopy, the use of ultrasound for spinal injection requires in-depth three-dimensional sono-anatomical knowledge of the spine region, to avoid misinterpretation of imagery [9]. Ultrasound has poor image quality for deeper structures such as the spinal cord, especially in obese patients, and is unable to penetrate bone. Deep vessels are difficult to identify on ultrasound, thus precluding visualization in instances of inadvertent intravascular medication injection.

This audit had several limitations. Firstly, the clinical audit was not designed to directly compare outcomes for ultrasound-guided injection against the conventional standard of care, which may include fluoroscopic or CT- guided injection. Second, the exact intervention time and quality of life measures were not collected as part of the center's routine clinical practice. These additional data would have been useful to objectively illustrate advantages in cost and procedural time with ultrasound spine injection. Third, as this was a new service, the sample size was relatively small. A larger dataset is required to confirm the efficacy of ultrasound-guided spine injection and the rate of adverse events. We were also unable to adjust for the confounding effects of concurrent oral analgesia use on pain and disability scores, due to the small sample size, though it should be noted that most of these patients were referred for spine injection after failure of conservative treatment (including pharmacotherapy). Finally, there were a minority of patients who did not respond to ultrasound-guided injection. A prospective study will hence be useful to determine clinical factors predicting successful ultrasound-guided spinal injection.

\section{Conclusions}

Ultrasound-guided spinal injection appears to be a safe, feasible, accurate, and cost-effective procedure for the treatment of LBP. It requires a mandatory learning curve to achieve good visualization and instillation of the injectate. Proper identification of pain generators and careful patient selection are crucial to achieving good functional outcomes.

\section{Conflict of Interest}

No potential conflict of interest relevant to this article was reported.

\section{Author Contributions}

Matthew Tay: drafted the paper, acquisition of data, analysis and interpretation of data; Shauna Christine Sim Hwei Sian: critical revision of the paper; Eow Chen Zhi: critical revision of the paper; Kelvin Lor Kah Ho: critical revision of the paper; Ong Joo Haw: critical revision of the paper; and Dinesh Sirisena: conception, design and critical revision of the paper.

\section{References}

1. Walker BF. The prevalence of low back pain: a sys- 
tematic review of the literature from 1966 to $1998 . J$ Spinal Disord 2000;13:205-17.

2. Last AR, Hulbert K. Chronic low back pain: evaluation and management. Am Fam Physician 2009;79:1067-74.

3. Schwarzer AC, Wang SC, Bogduk N, McNaught PJ, Laurent R. Prevalence and clinical features of lumbar zygapophysial joint pain: a study in an Australian population with chronic low back pain. Ann Rheum Dis 1995;54:100-6.

4. Manchikanti L, Boswell MV, Singh V, Pampati V, Damron KS, Beyer CD. Prevalence of facet joint pain in chronic spinal pain of cervical, thoracic, and lumbar regions. BMC Musculoskelet Disord 2004;5:15.

5. Baron R, Binder A, Attal N, Casale R, Dickenson $\mathrm{AH}$, Treede RD. Neuropathic low back pain in clinical practice. Eur J Pain 2016;20:861-73.

6. Cohen SP, Chen Y, Neufeld NJ. Sacroiliac joint pain: a comprehensive review of epidemiology, diagnosis and treatment. Expert Rev Neurother 2013;13:99116.

7. Kaye AD, Manchikanti L, Abdi S, et al. Efficacy of epidural injections in managing chronic spinal pain: a best evidence synthesis. Pain Physician 2015;18:E939-1004.

8. Ye L, Wen C, Liu H. Ultrasound-guided versus low dose computed tomography scanning guidance for lumbar facet joint injections: same accuracy and efficiency. BMC Anesthesiol 2018;18:160.

9. Chi M, Chen AS. Ultrasound for lumbar spinal procedures. Phys Med Rehabil Clin N Am 2018;29:4960.

10. Greher M, Scharbert G, Kamolz LP, et al. Ultrasoundguided lumbar facet nerve block: a sonoanatomic study of a new methodologic approach. Anesthesiology 2004;100:1242-8.

11. Loizides A, Peer S, Plaikner M, et al. Ultrasoundguided injections in the lumbar spine. Med Ultrason 2011;13:54-8.

12. Park Y, Lee JH, Park KD, Ahn JK, Park J, Jee H. Ultrasound-guided vs. fluoroscopy-guided caudal epidural steroid injection for the treatment of unilateral lower lumbar radicular pain: a prospective, randomized, single-blind clinical study. Am J Phys Med Rehabil 2013;92:575-86.

13. Evansa I, Logina I, Vanags I, Borgeat A. Ultrasound versus fluoroscopic-guided epidural steroid injec- tions in patients with degenerative spinal diseases: a randomised study. Eur J Anaesthesiol 2015;32:262-8.

14. Hashemi M, Dadkhah P, Taheri M, Haji Seyed Abootorabi SM, Naderi-Nabi B. Ultrasound-guided lumbar transforaminal epidural injections; a single center fluoroscopic validation study. Bull Emerg Trauma 2019;7:251-5.

15. Kumar A, Sinha C, Bhadani UK, Sharma S. Ultrasound-guided lumbar transforaminal injection through interfacet approach. Saudi J Anaesth 2018;12:169-70.

16. Han SH, Park KD, Cho KR, Park Y. Ultrasound versus fluoroscopy-guided medial branch block for the treatment of lower lumbar facet joint pain: a retrospective comparative study. Medicine (Baltimore) 2017;96:e6655.

17. Robin F, Coiffier G, Albert JD, et al. Is ultrasoundguided caudal steroid injection effective in the management of lower lumbar radicular pain?: a two-center prospective observational study on 150 patients. Joint Bone Spine 2019:S1297-319X(19)30172-1.

18. Ha DH, Shim DM, Kim TK, Kim YM, Choi SS. Comparison of ultrasonography- and fluoroscopy-guided facet joint block in the lumbar spine. Asian Spine J 2010;4:15-22.

19. Yun DH, Kim HS, Yoo SD, et al. Efficacy of ultrasonography-guided injections in patients with facet syndrome of the low lumbar spine. Ann Rehabil Med 2012;36:66-71.

20. Galiano K, Obwegeser AA, Bodner G, et al. Ultrasound guidance for facet joint injections in the lumbar spine: a computed tomography-controlled feasibility study. Anesth Analg 2005;101:579-83.

21. Fukui S, Ohseto K, Shiotani M, Ohno K, Karasawa H, Naganuma Y. Distribution of referred pain from the lumbar zygapophyseal joints and dorsal rami. Clin J Pain 1997;13:303-7.

22. Farrar JT, Young JP Jr, LaMoreaux L, Werth JL, Poole RM. Clinical importance of changes in chronic pain intensity measured on an 11-point numerical pain rating scale. Pain 2001;94:149-58.

23. Fairbank JC, Pynsent PB. The Oswestry Disability Index. Spine (Phila Pa 1976) 2000;25:2940-52.

24. Yoon SH, O’Brien SL, Tran M. Ultrasound guided spine injections: advancement over fluoroscopic guidance? Curr Phys Med Rehabil Rep 2013;1:10413. 
25. Yang G, Liu J, Ma L, et al. Ultrasound-guided versus fluoroscopy-controlled lumbar transforaminal epidural injections: a prospective randomized clinical trial. Clin J Pain 2016;32:103-8.

26. Carey TS, Garrett JM. The relation of race to outcomes and the use of health care services for acute low back pain. Spine (Phila Pa 1976) 2003;28:390-4.
27. Hofmeister M, Dowsett LE, Lorenzetti DL, Clement F. Ultrasound- versus fluoroscopy-guided injections in the lower back for the management of pain: a systematic review. Eur Radiol 2019;29:3401-9.

28. Allegri M, Montella S, Salici F, et al. Mechanisms of low back pain: a guide for diagnosis and therapy: version 2. F1000Res 2016;5:F1000 Faculty Rev-1530. 